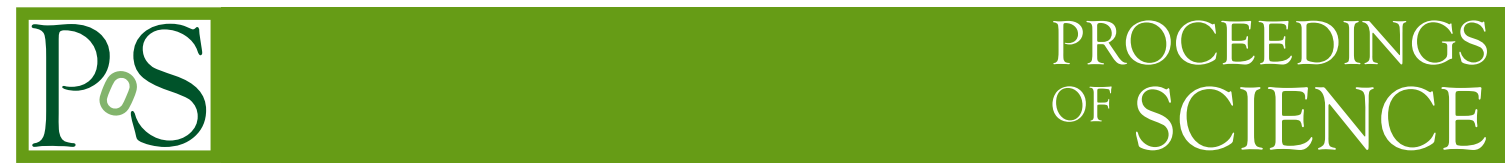

\title{
EMC effect: Past, Present, and Future
}

\author{
Nadia Fomin* \\ University of Tennessee \\ E-mail: nfomineutk.edu
}

Since the discovery of the EMC effect over 30 years ago, it has been of great theoretical interest and studied in several experimental measurements. No unified picture arose to explain the underlying cause of per nucleon structure function modification in nuclei. Precise measurements on light nuclei from JLab's $6 \mathrm{GeV}$ era revitalized this research by showing that traditional A or density dependent models of this nuclear modification do not work. The measurements will be reviewed, discussed and preliminary data on heavy targets from JLab's E03-103 will be presented.

XXIII International Workshop on Deep-Inelastic Scattering,

27 April - May 12015

Dallas, Texas

${ }^{*}$ Speaker. 


\section{Introduction}

Modification of quark momentum distributions in nuclei first observed by the EMC collaboration [1] in the early ' 80 s was a surprising result and has been a subject of great interest to both theorists and experimentalists, resulting in detailed follow-up measurements. However, in the 25 years following the original observation that nuclear structure functions are not just given by $N \times F_{2}^{N}+Z \times F_{2}^{P}$ - the sum of proton and neutron contributions of the constituents, no consensus was reached as to what underlying mechanism was responsible for this fact. Fig. 1 shows the unexpected depletion in the strength of quark distributions in carbon compared to those in the deuteron for the $0.3 \lesssim x \lesssim 0.7$ region. This shape appears to be universal across nuclei with the magnitude of the "dip" growing with $A$.

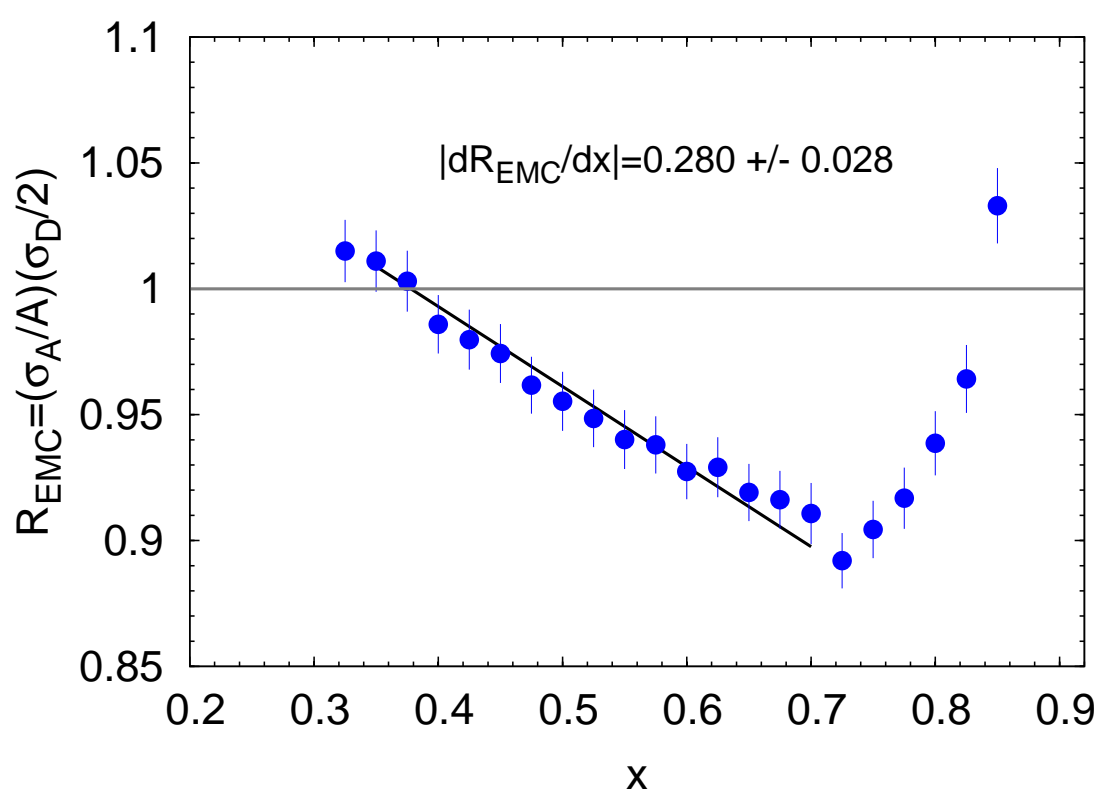

Figure 1: EMC ratio of per-nucleon carbon and deuteron cross sections as a function of $x$. The slope in the $0.3 \lesssim x \lesssim 0.7$ region is take to be the "size" of the EMC effect.

The physics models put forth to explain this observation have historically fallen into one of two classes. One focuses on using convolution models with binding effects, or detailed models of nucleon momentum distributions, or pion-exchange contributions - established nuclear effects. The second class suggests more exotic explanations such as 6- or 9-quark bags, "nuclear swelling" or similar modifications of the internal nucleon structure, as well as other possibilities $[2,3,4]$. At the same time, very simple models using the size of the nucleus (average nucleon density or simply the number of nucleons) seemed to describe the data trends seen. The results of Jlab E03-103 [5] breathed new life into this research, as simple $A$ - or density- dependent models were no longer good explanations for the data.

Cross section measurements were performed on ${ }^{2} \mathrm{H},{ }^{3} \mathrm{He},{ }^{4} \mathrm{He},{ }^{9} \mathrm{Be}$, and ${ }^{12} \mathrm{C}$ targets. The size of the EMC effect $\left(d R_{E M C} / d x\right)$ was taken to be the slope of the $A / D$ nuclear structure function ratio for $0.3 \leq x \leq 0.7$, rather than the $A / D$ ratio at $x=0.6$, as was done with past analyses. Using the slope makes one insensitive to normalization corrections. Jlab E03-103 [5] provided the most 
precise measurements of these ratios for light targets, and showed that the EMC effect cannot be explained with simple density dependence model, as is shown in the left panel of Fig. 3.

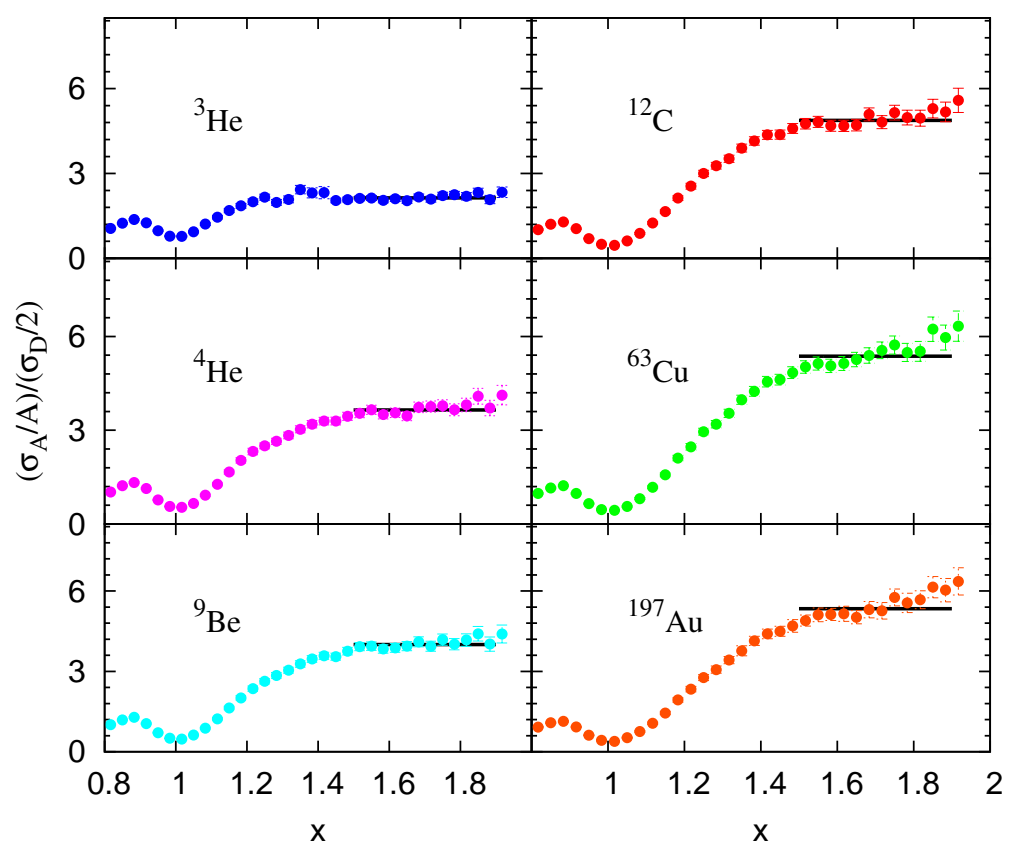

Figure 2: The six-panel plot shows per-nucleon cross section ratios for a variety of target nuclei as a function of $x$. The ratio $a_{2}$ was taken in the $1.5 \lesssim x \lesssim 1.9$ region.

\section{Connection with correlated NN pairs}

On the other side of the kinematic spectrum lie measurements that aim to access the momentum distributions of nucleons inside nuclei. These distributions are not a physics observable and instead, electron scattering is used to measure differential cross sections at $x_{\text {bjorken }}>1$. This kinematic region is accessible only because bound nucleons can share momentum and reach velocities unavailable to free nucleons. The $x>1$ kinematic region is dominated by the high-momentum tail $\left(k>k_{f e r m i}\right)$ of the distribution, and extends beyond what is expected in mean field models. Nucleons with momenta $k>k_{\text {fermi }}$ are believed to have their origins in multi-nucleon correlations, where inter-nucleon separation can get smaller than $1 \mathrm{fm}$, leading to hard-core interactions. Inside the deuteron, only the $n p$ configuration is available, so the cross section strength seen for $1.4 \lesssim x \lesssim 2$ reflects the presence of $n p$ pairs. If all high-momentum nucleons are born in these NN correlations, then the high-momentum tails for $A>2$ nuclei will, to a first approximation, be rescaled versions of the deuteron. Taking the cross section ratios in this region, we should then see a scaling plateau whose magnitude will yield the relative number of $n p$ pairs. The $n p$ dominance of $2 \mathrm{~N}$ pairs in $A>2$ nuclei was established in nucleon knockout $[6,7]$ experiments at JLab and BNL. These plateaus are exactly what is seen in the data $[8,9,10,11]$. An example of the plateaus from E02-019 is shown in Fig. 2, and their magnitude is designated as $a_{2}$.

The interesting and somewhat unexpected observation is that the SRC plateaus exhibit the same deviation from a simple density dependence as we see in the EMC effect, shown in Fig. 3. 

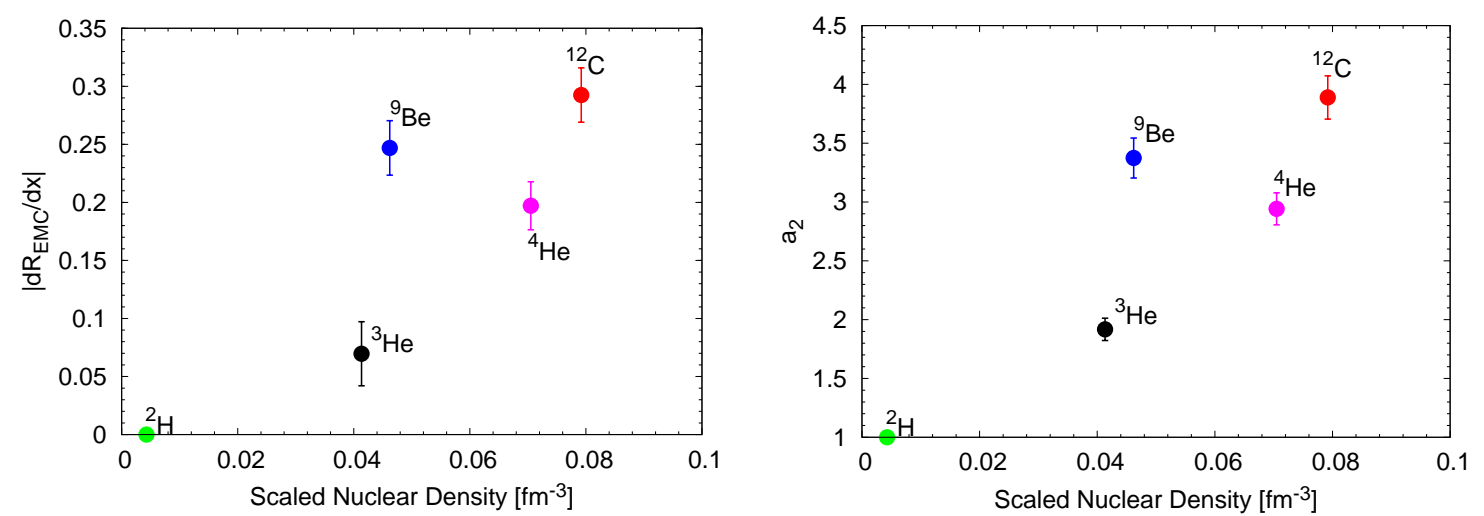

Figure 3: The figure on the left shows the size of the EMC effect, taken to be the slope of the $A / D$ nuclear structure function ratio for $0.3 \leq x \leq 0.7$. The figure on the right shows relative contribution of SRCs, i.e. the A/D cross section ratio for $x>1\left(a_{2}\right)$. Both are plotted as a function of scaled nuclear density, where the scaling factor is $(A-1) / A$ to account for the fact that the nucleon being probed only sees the density due to the remaining $A-1$ nucleons. Only light nuclei are shown to highlight the common deviation of ${ }^{9} \mathrm{Be}$ from a simple density dependent picture.

While not conclusive yet, it certainly suggests a connection between the phenomena and merits further investigation.

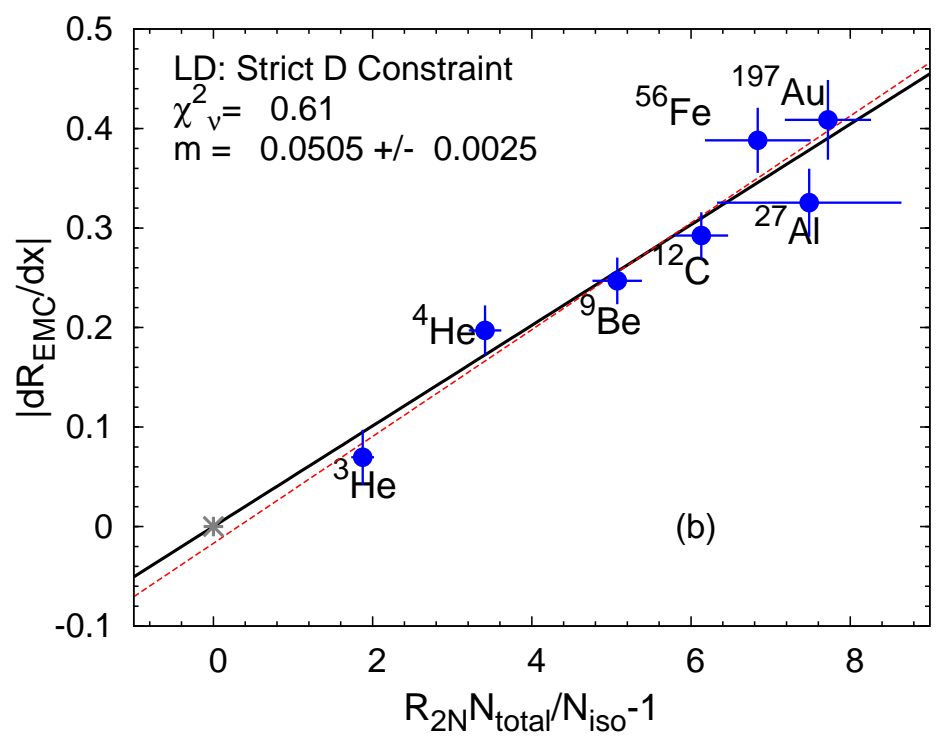

Figure 4: The size of the EMC effect as a function of number of $n p$ short-range pairs relative to the deuteron $\left(R_{2 N}\right.$ is derived from $\left.a_{2}\right)$. Since we believe the EMC effect probes the entire nucleus, the latter quantity has been scaled to the total number of possible $\mathrm{NN}$ pairs $\left(\mathrm{N}_{\text {total }}\right)$, which yields an improved correlation.

Recent analyses $[12,13,14]$ show an excellent linear correlation between the two phenomena, even though the EMC effect probes quark distributions and SRC ratios look at short-range configurations via electrons scattering from high-momentum nucleons. This relationship is displayed in Fig. 4, where quantity on the $\mathrm{x}$-axis is derived from the SRC ratios to give the total number 
of NN pairs, rather than just $n p$ ones. This difference is a small one, but the underlying cause of the correlation is different: is the modification of the quark distributions related simply to the number of high-momentum nucleons (raw SRC ratios) or driven by local nucleon density (represented by all NN correlations)? Existing data do not allow us to draw meaningful conclusions yet. It's unclear whether the correlation relationship is strictly accidental or if the measurements of high-momentum nucleons (and the short-range configurations where they are born) reveal the mechanism for the structure function modification seen in EMC effect results. Precise data with light nuclei can shed light on this intriguing question, just as the new ${ }^{9} \mathrm{Be}$ data caused us to discard decades-old models of $A$ and simple density dependence.

\section{Future measurements}

As was illustrated in Fig. 3, an interesting and unexpected feature of the EMC effect was uncovered by clever choices of targets. It suggests that light targets might allow for sensitive tests of the nuclear dynamics. Upcoming Jlab experiment E12-10-108 will continue the study of the EMC effect at higher $\mathrm{Q}^{2}$ values made accessible by the $12 \mathrm{GeV}$ upgrade. Additionally, the search will focus on light nuclei as well as signs of a possible isospin dependence. With these considerations in mind, experimental targets include ${ }^{3} \mathrm{He},{ }^{4} \mathrm{He},{ }^{6} \mathrm{Li},{ }^{7} \mathrm{Li},{ }^{9} \mathrm{Be},{ }^{10} \mathrm{~B},{ }^{11} \mathrm{~B},{ }^{12} \mathrm{C},{ }^{40} \mathrm{Ca}$, ${ }^{48} \mathrm{Ca}$, and ${ }^{63} \mathrm{Cu}$. A complementary measurement of SRC ratios with the same targets will allow for ongoing tests of the connection between the two physics phenomena.

While the light ${ }^{9} \mathrm{Be}$ nucleus is responsible for a lot of the resurgence of interest in the EMC effect, another place to look for hints of the underlying cause might be high asymmetric nuclei $(\mathrm{N} \neq \mathrm{Z})$. In nuclei with a neutron excess, protons are more likely to participate in $n p$ correlations than neutrons are. If the connection to the EMC effect is real, this implies larger modification of $u$-quark distributions than that for $d$-quarks.

It is also possible to probe the flavor dependence of the EMC effect through parity-violating DIS $[16,17]$. A measurement complementary to traditional electromagnetic interaction ones, this would allow studies of possible isovector behavior. PVDIS probes the effective weak couplings through an interference of the neutral $Z$ boson and the photon, and measurements on heavy $N \neq Z$ nuclei can elucidate new information. Such experiments are possible at Jefferson Lab with existing apparatus.

\section{Summary}

For almost 30 years after the EMC collaboration first observed nuclear modification of the quark distributions, the underlying case has remained largely a mystery. Recent measurements have given us hints of where we should focus our experimental and theoretical efforts by showing that naive density and A-dependent pictures don't work, but that there might be a connection to NN correlations, a seemingly different kind of physics phenomena. Upcoming experiments at Jefferson Lab should provide new clues to this three decade old puzzle.

\section{References}

[1] J.J. Aubert et al. Phys. Lett., B123:275, 1983. 
[2] D. F. Geesaman, K. Saito, and A. W. Thomas. The nuclear emc effect. Ann. Rev. Nucl. Sci., 45:337-390, 1995.

[3] P. R. Norton. The emc effect. Rept. Prog. Phys., 66:1253-1297, 2003.

[4] Gunther Piller and Wolfram Weise. Nuclear deep inelastic lepton scattering and coherence phenomena. Phys. Rept., 330:1-94, 2000.

[5] J. Seely et al. New measurements of the EMC effect in very light nuclei. Phys. Rev. Lett., 103:202301, 2009.

[6] R. Subedi et al. Probing Cold Dense Nuclear Matter. Science, 320:1476-1478, 2008.

[7] E. Piasetzky, M. Sargsian, L. Frankfurt, M. Strikman, and J.W. Watson. Evidence for the strong dominance of proton-neutron correlations in nuclei. Phys. Rev. Lett., 97:162504, 2006.

[8] L. L. Frankfurt, M. I. Strikman, D. B. Day, and M. Sargsian. Evidence for short range correlations from high $q^{2}$ (e, e') reactions. Phys. Rev. C, 48:2451-2461, 1993.

[9] K. .S. Egiyan et al. Observation of nuclear scaling in the $a\left(e, e^{\prime}\right)$ reaction at $x_{b}>1$. Phys. Rev. C, 68:014313, 2003.

[10] K. S. Egiyan et al. Measurement of 2- and 3-nucleon short range correlation probabilities in nuclei. Phys. Rev. Lett., 96:082501, 2006.

[11] N. Fomin et al. New measurements of high-momentum nucleons and short-range structures in nuclei. Phys. Rev. Lett. , 108:092502, 2012.

[12] L.B. Weinstein, E. Piasetzky, D.W. Higinbotham, J. Gomez, O. Hen, and R. Shneor. Short Range Correlations and the EMC Effect. Phys. Rev. Lett., 106:052301, 2011.

[13] O. Hen, E. Piasetzky and L. B. Weinstein, Phys. Rev. C 85, 047301 (2012).

[14] J. Arrington, A. Daniel, D. Day, N. Fomin, D. Gaskell, and P. Solvignon. A detailed study of the nuclear dependence of the emc effect and short-range correlations. Phys. ReV. C, 86:065204, 2012.

[15] A. Daniel, J. Arrington and D. Gaskell, JLab Proposal PR12-10-108

[16] S. Riordan and R. Beminiwattha et al., JLab Proposal PR12-14-007

[17] I. C. Cloet, W. Bentz and A. W. Thomas, Phys. Rev. Lett. 109, 182301 (2012). 